\title{
Prognostic impact of estimated remnant- like particle cholesterol in patients with differing glycometabolic status: an observational cohort study from China
}

Qi Zhao ${ }^{1,2}$, Ting-Yu Zhang ${ }^{1,2}$, Yu-Jing Cheng ${ }^{1,2}$, Yue Ma ${ }^{3}$, Ying-Kai Xu ${ }^{1,2}$, Jia-Qi Yang ${ }^{1,2}$ and Yu-Jie Zhou ${ }^{1,2^{*}}$

\begin{abstract}
Background: It is uncertain whether estimated remnant-like particle cholesterol (RLP-C) could predict residual risk in patients with different glycometabolic status. This study aimed to evaluate the relationship between estimated RLP-C and adverse prognosis in patients with non-ST-segment elevation acute coronary syndrome (NSTE-ACS) treated with percutaneous coronary intervention $(\mathrm{PCl})$ and to identify the potential impact of glycometabolism on the predictive value of estimated RLP-C.

Methods: The study assessed 2419 participants with NSTE-ACS undergoing PCI at Beijing Anzhen Hospital from January to December 2015. Estimated RLP-C was calculated as follows: total cholesterol (TC) minus low-density lipoprotein cholesterol (LDL-C) and high-density lipoprotein cholesterol (HDL-C). The adverse events included allcause death, non-fatal myocardial infarction (MI), and ischemia-driven revascularization.

Results: Estimated RLP-C was prominently associated with adverse prognosis in the total population [hazard ratio (HR) 1.291 per 1-SD increase, 95\% confidence interval (Cl) 1.119-1.490, $P<0.001$ ], independent of confounding risk factors. However, subgroup analysis showed that increasing estimated RLP-C was related to a higher risk of adverse events in the diabetic population only [HR 1.385 per 1-SD increase, 95\% Cl 1.183-1.620, $P<0.001]$. Estimated RLP-C failed to be a significant determinant of adverse prognosis in non-diabetic and pre-diabetic subgroups. The addition of estimated RLP-C to a baseline model including traditional risk factors enhanced the predictive performance both in total and diabetic populations.
\end{abstract}

Conclusions: High estimated RLP-C level is a significant predictor for recurrent adverse events in patients with diabetes and NSTE-ACS treated with PCI.

Keywords: Remnant-like particle cholesterol, Non-ST-segment elevation acute coronary syndrome, Percutaneous coronary intervention

\footnotetext{
* Correspondence: azzyj12@163.com

'Department of Cardiology, Beijing Anzhen Hospital, Capital Medical University, Beijing 100029, China

${ }^{2}$ Beijing Institute of Heart Lung and Blood Vessel Disease, Beijing Key Laboratory of Precision Medicine of Coronary Atherosclerotic Disease, Clinical center for coronary heart disease, Capital Medical University, Beijing 100029,

China

Full list of author information is available at the end of the article
}

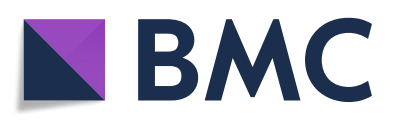

( ) The Author(s). 2020 Open Access This article is licensed under a Creative Commons Attribution 4.0 International License, which permits use, sharing, adaptation, distribution and reproduction in any medium or format, as long as you give appropriate credit to the original author(s) and the source, provide a link to the Creative Commons licence, and indicate if changes were made. The images or other third party material in this article are included in the article's Creative Commons licence, unless indicated otherwise in a credit line to the material. If material is not included in the article's Creative Commons licence and your intended use is not permitted by statutory regulation or exceeds the permitted use, you will need to obtain permission directly from the copyright holder. To view a copy of this licence, visit http://creativecommons.org/licenses/by/4.0/. The Creative Commons Public Domain Dedication waiver (http://creativecommons.org/publicdomain/zero/1.0/) applies to the data made available in this article, unless otherwise stated in a credit line to the data. 


\section{Background}

As the most serious manifestation of atherosclerotic cardiovascular disease (ASCVD), acute coronary syndrome (ACS) leads to a consistently higher risk of recurrence of cardiovascular outcomes, despite wide application of evidence-based secondary prevention strategies [1, 2]. Low-density lipoprotein cholesterol (LDL-C) has been extensively recognized as the significant risk factor for ASCVD, reduction of which is an effective therapy to reduce cardiovascular risks [3]. Despite regulating LDL-C with guideline-recommended therapies, patients with ACS remain at a higher risk of recurrent cardiovascular outcomes [4-7], which indicates that there are factors other than LDL-C that determine risk.

Studies have reported that the residual risk can be partly ascribed to an increased level of remnant lipoproteins $[2,4,8,9]$. Remnant lipoproteins are lipoproteins that are rich in triglycerides (TGs), components of which include chylomicron remnant, very-low-density lipoprotein (VLDL), and intermediate-density lipoprotein (IDL) [10]. The cholesterol content of remnant lipoproteins is defined as remnant-like particle cholesterol (RLP-C). Nowadays, the pattern of targeting LDL-C alone has changed, with recent guidelines highlighting the importance of non-high-density lipoprotein cholesterol (nonHDL-C), which includes RLP-C, on the pathogenesis of atherosclerosis and thus its availability as an additional therapeutic target [11]. As a component of non-HDL-C, it is of great significance to further clarify the effect of RLP-C on coronary atherosclerosis.

The prognostic significance of RLP-C on adverse prognosis in the specific cohort diagnosed with non-STsegment elevation acute coronary syndrome (NSTEACS) and treated with percutaneous coronary intervention (PCI) were not explicitly investigated. Results from former studies revealed that the prognostic impact of RLP-C seems to be more prominent in patients with metabolic syndrome or type 2 diabetes [12-16]. It is worth exploring whether the prognostic value of RLP-C varies among populations with different glycometabolic status. The current study is the first to investigate the relationship between estimated RLP-C and recurrent adverse events, and confirm the potential impact of glycometabolic status on the predictive value of estimated RLP-C in participants with NSTE-ACS undergoing PCI.

\section{Methods}

\section{Study population}

This study retrospectively screened patients diagnosed with NSTE-ACS who received PCI treatment in Beijing Anzhen Hospital (Beijing, China) from January to December 2015. The definition of NSTE-ACS was in accordance with corresponding guidelines [17], including non-ST segment elevation myocardial infarction (NSTEMI) and unstable angina pectoris (UA). The exclusion criteria included: (1) missing clinical, laboratory, and/or angiographic data; (2) history of cardiogenic shock, chronic inflammatory disease, or neoplasm; (3) evidence of active infection; (4) severe renal dysfunction with estimated glomerular filtration rate (eGFR) lower than $30 \mathrm{~mL} /\left(\mathrm{min}^{*} 1.73 \mathrm{~m}^{2}\right)$, severe hepatic disease, and other serious diseases; (5) death in hospital, complications or procedure failure of PCI. Based on the enrollment criteria, 2419 participants were finally included in the study.

The study population was divided into three subgroups according to the glycometabolic status. Patients with a definite prior diagnosis of diabetes and glycosylated hemoglobin $\mathrm{A} 1 \mathrm{c}(\mathrm{HbA} 1 \mathrm{c}) \geq 6.5 \%$ on admission were defined as the diabetic population. Patients with HbA1c level $<5.7$ and $5.7 \% \sim 6.4 \%$ were considered as nondiabetic and pre-diabetic population, respectively, as previous guidelines mentioned [18].

\section{Data collection}

The enrolled patients' demographic characteristics, clinical information, laboratory investigations, and coronary procedural results were retrieved and collected by using the medical information recording system from Beijing Anzhen Hospital.

All laboratory parameters were analyzed in the central laboratory of the hospital by using the first fasting venous blood samples before the baseline PCI. High-sensitivity Creactive protein (hs-CRP), creatinine, uric acid, fasting blood glucose (FBG), and HbA1c were measured by standard methods. Concentrations of TGs, total cholesterol (TC), and high-density lipoprotein cholesterol (HDLC) were quantified by standard enzymatic methods. LDL$\mathrm{C}$ was determined by the homogeneous direct method. Estimated RLP-C was calculated by subtracting LDL-C and HDL-C from TC, which was recommended by relevant dyslipidemia guidelines $[19,20]$. The eGFR was computed by MDRD equation as previously proposed [21]. Two-dimensional Simpson's method was applied to evaluate the left ventricular ejection fraction (LVEF).

Coronary angiographic data were analyzed and evaluated by visual measurements, and the results were documented and verified by at least two experienced cardiologists. The multi-vessel disease was defined as more than two main epicardial coronary arteries with stenosis $\geq 50 \%$. The lesion with complete cessation of blood flow [thrombolysis in myocardial infarction (TIMI) flow grade 0] and duration $\geq 3$ months was considered as a chronic total occlusion. An independent lesion with a length $\geq 20 \mathrm{~mm}$ was defined as a diffuse lesion, and the lesion implicating the origin of an important side branch was defined as a bifurcation lesion. Coronary procedures were carried out based on the relevant guideline of China [22], and specific procedure strategies were selected by practiced cardiologists. 


\section{Follow-up}

After coronary procedures had been performed, all participants received routine follow-up at 3, 6, and 12 months and then annually until 36 months by means of phone interviews with the patient and/or family members. Corresponding medical records were referred to verify the authenticity in case that ambiguous information was obtained. Adverse outcomes including all-cause death, non-fatal myocardial infarction (MI), and ischemia-driven revascularization were recorded as composite or separate adverse event to implement the current analysis. If participants experienced more than one adverse event during the 36-month follow-up, only the first instance of the event was selected to proceed the current study.

\section{Statistical analysis}

Continuous variables were displayed as mean \pm SD or median (25th and 75th percentiles), and compared by $\mathrm{t}$ test or Mann-Whitney U test, as appropriate. Nominal variables were expressed as absolute quantities and proportions, and compared by $\chi^{2}$ test or Fisher's exact test when appropriate. The correlations between estimated RLP-C and other variables were assessed by Pearson or Spearman's rank correlation test as applicable. The incidence of events in groups with lower and higher median of estimated RLP-C was described by Kaplan-Meier curves, and the difference between groups was compared by log-rank test. The simple Cox analyses were primarily conducted to confirm the significant predictors of adverse events. The variables with statistical significance $(P<0.05)$ in simple Cox analysis were analyzed with multiple Cox analysis to investigate the independent determinants of adverse events. The results of Cox analysis were interpreted using hazard ratio (HR) and 95\% confidence intervals $(\mathrm{CI})$. The HR was examined by $1-\mathrm{SD}$ change in continuous variables except for age, heart rate, systolic blood pressure (SBP), and number of stents. Cstatistics that consisted of receiver-operating characteristic (ROC) analysis was applied to estimate the additional discriminative ability of estimated RLP-C for predicting worse outcomes on the basis of the baseline model including traditional risk factors. Differences between the area under the ROC curve (AUC) of various models were compared by DeLong's test. Moreover, the incremental reclassification and discrimination ability of estimated RLP-C beyond the baseline model for predicting adverse events was further determined by category-free net reclassification improvement (NRI) and integrated discrimination improvement (IDI). The population was divided into three subgroups according to glycometabolic status: diabetic, pre-diabetic, and non-diabetic groups. Similar statistical analyses were performed for each subgroup. Statistical analyses were performed using SPSS (version 23.0; IBM, IL, USA), MedCalc Statistical
Software (version 19.1; Ostend, Belgium), and the $\mathrm{R}$ Project (version 3.5.1). A $P$ value of 0.05 was applied to assess statistical significance.

\section{Results \\ Baseline characteristics}

The final enrolled 2419 participants (age 60.08 \pm 8.97 ; $71.8 \%$ male) were divided into with-event and withoutevent group, baseline characteristics of which were summarized in Table 1. The level of estimated RLP-C in participants with an adverse event was prominently higher than those without $(0.90 \pm 0.61$ vs. $0.65 \pm 0.35, P<0.001)$. Patients with an adverse event were observed to be older and had higher body mass index (BMI), heart rate, and SBP. The prevalence of prior MI, PCI, coronary artery bypass grafting (CABG), stroke, and diabetes were higher in the group with an event. With regard to laboratory parameters, participants that developed adverse events displayed higher TGs, TC, hs-CRP, creatinine, HbA1c, and FBG, but lower HDL-C, eGFR, and LVEF. In terms of the angiographic information, more complex coronary artery lesions were exhibited and more stents were implanted in participants with an adverse event. In addition, more participants were diagnosed with NSTEMI, and more angiotensin-converting enzyme inhibitors (ACEI), oral hypoglycemic agents, and insulin were prescribed in patients with an adverse event.

Estimated RLP-C was higher in participants with diabetes than pre-diabetes $(0.74 \pm 0.51$ vs $0.68 \pm 0.36, P=$ $0.003)$ and non-diabetes $(0.74 \pm 0.51$ vs $0.66 \pm 0.37$, $P<0.001)$. However, the difference between pre-diabetic and non-diabetic populations was not conspicuous $(0.68 \pm 0.36$ vs $0.66 \pm 0.37, P=0.339)$ (Fig. 1$)$. Estimated RLP-C was positively correlated to TGs $(r=0.853$, $P<0.001)$, TC $(r=0.455, P<0.001)$, and LDL-C $(r=$ $0.112, P<0.001)$, while negatively correlated to HDL-C $(r=-0.173, P<0.001)$.

\section{Predictive value of estimated RLP-C in total population}

During the follow-up period, 454 (18.8\%) patients experienced an adverse event, which comprised of 21 (0.9\%) all-cause deaths, 117 (4.8\%) non-fatal MI, and 316 (13.1\%) of ischemia-driven revascularization. KaplanMeier curves for the incidence of the composite and each component of adverse events were displayed in Fig. 2. Compared with a lower median of estimated RLP$C$, patients with a higher median presented with a higher incidence of composite adverse events $(P<0.001)$, nonfatal MI $(P=0.003)$, and ischemia-driven revascularization $(P<0.001)$ (Fig. 2). However, there was no difference on incidence of all-cause death between groups $(P=0.260)$ (Fig. 2).

Multiple Cox analysis adjusted for variables that were significant $(P<0.05)$ in simple Cox analysis (shown in 
Table 1 Baseline clinical characteristics of the study population

\begin{tabular}{|c|c|c|c|c|}
\hline & Total population, $n=2419$ & Without event, $n=1965$ & With event, $n=454$ & $P$ \\
\hline Age, years & $60.08 \pm 8.97$ & $59.60 \pm 8.72$ & $62.16 \pm 9.70$ & $<0.001$ \\
\hline Male, n (\%) & $1737(71.8)$ & $1422(72.4)$ & $315(69.4)$ & 0.203 \\
\hline $\mathrm{BMI}, \mathrm{kg} / \mathrm{m}^{2}$ & $26.21 \pm 3.45$ & $26.13 \pm 3.40$ & $26.55 \pm 3.61$ & 0.019 \\
\hline Heart rate, bpm & $69.77 \pm 10.15$ & $69.44 \pm 10.00$ & $71.17 \pm 10.69$ & 0.002 \\
\hline $\mathrm{SBP}, \mathrm{mmHg}$ & $130.30 \pm 16.52$ & $129.80 \pm 15.99$ & $132.44 \pm 18.50$ & 0.005 \\
\hline $\mathrm{DBP}, \mathrm{mmHg}$ & $77.05 \pm 9.90$ & $77.00 \pm 9.68$ & $77.25 \pm 10.80$ & 0.661 \\
\hline Smoking, n (\%) & $1381(57.1)$ & $1127(57.4)$ & $254(55.9)$ & 0.585 \\
\hline Drinking, n (\%) & $562(23.2)$ & $468(23.8)$ & $94(20.7)$ & 0.157 \\
\hline Family history of CAD, n (\%) & $254(10.5)$ & $203(10.3)$ & $51(11.2)$ & 0.572 \\
\hline \multicolumn{5}{|l|}{ Medical history, n (\%) } \\
\hline Hypertension & $1511(62.5)$ & $1210(61.6)$ & $301(66.3)$ & 0.061 \\
\hline Prior Ml & $527(21.8)$ & $348(17.7)$ & 179 (39.4) & $<0.001$ \\
\hline Prior PCl & $414(17.1)$ & $280(14.2)$ & $134(29.5)$ & $<0.001$ \\
\hline Prior CABG & $55(2.3)$ & $23(1.2)$ & $32(7.0)$ & $<0.001$ \\
\hline Prior stroke & 281 (11.6) & $204(10.4)$ & $77(17.0)$ & $<0.001$ \\
\hline Prior PAD & $84(3.5)$ & $63(3.2)$ & $21(4.6)$ & 0.137 \\
\hline \multicolumn{5}{|l|}{ Glycometabolic status } \\
\hline Non-diabetes & $926(38.3)$ & $829(42.2)$ & $97(21.4)$ & $<0.001$ \\
\hline Pre-diabetes & $645(26.7)$ & $531(27.0)$ & $114(25.1)$ & 0.406 \\
\hline Diabetes & $848(35.1)$ & $605(30.8)$ & $243(53.5)$ & $<0.001$ \\
\hline \multicolumn{5}{|l|}{ Laboratory results } \\
\hline TGs, mmol/L & $1.84 \pm 1.32$ & $1.69 \pm 1.05$ & $2.47 \pm 2.00$ & $<0.001$ \\
\hline $\mathrm{TC}, \mathrm{mmol} / \mathrm{L}$ & $4.17 \pm 1.06$ & $4.14 \pm 1.05$ & $4.33 \pm 1.07$ & 0.001 \\
\hline $\mathrm{LDL}-\mathrm{C}, \mathrm{mmol} / \mathrm{L}$ & $2.50 \pm 0.88$ & $2.50 \pm 0.89$ & $2.50 \pm 0.85$ & 0.962 \\
\hline $\mathrm{HDL}-\mathrm{C}, \mathrm{mmol} / \mathrm{L}$ & $0.98 \pm 0.23$ & $0.99 \pm 0.24$ & $0.92 \pm 0.21$ & $<0.001$ \\
\hline Estimated RLP-C, mmol/L & $0.69 \pm 0.42$ & $0.65 \pm 0.35$ & $0.90 \pm 0.61$ & $<0.001$ \\
\hline hs-CRP, mg/L & $1.29(0.58,3.31)$ & $1.22(0.53,3.06)$ & $1.87(0.77,4.29)$ & $<0.001$ \\
\hline Creatinine, $\mu \mathrm{mol} / \mathrm{L}$ & $76.00 \pm 16.95$ & $75.68 \pm 16.49$ & $77.42 \pm 18.76$ & 0.048 \\
\hline eGFR, ml/(min*1.73m²) & $93.49 \pm 20.36$ & $94.09 \pm 20.11$ & $90.91 \pm 21.22$ & 0.003 \\
\hline Uric acid, $\mu \mathrm{mol} / \mathrm{L}$ & $346.22 \pm 82.64$ & $346.45 \pm 81.45$ & $345.21 \pm 87.69$ & 0.774 \\
\hline FBG, mmol/L & $6.20 \pm 1.94$ & $6.01 \pm 1.71$ & $7.03 \pm 2.57$ & $<0.001$ \\
\hline $\mathrm{HbA} 1 \mathrm{c}, \%$ & $5.90(5.50,6.60)$ & $5.80(5.50,6.40)$ & $6.40(5.80,8.00)$ & $<0.001$ \\
\hline LVEF, \% & $65.00(60.00,68.00)$ & $65.00(61.00,69.00)$ & $63.00(57.00,67.00)$ & $<0.001$ \\
\hline Initial diagnosis, n (\%) & & & & 0.001 \\
\hline UA & $2018(83.4)$ & $1662(84.6)$ & $356(78.4)$ & \\
\hline NSTEMI & 401 (16.6) & $303(15.4)$ & $98(21.6)$ & \\
\hline \multicolumn{5}{|l|}{ Medical treatment, n (\%) } \\
\hline ACEI & $734(30.3)$ & $577(29.4)$ & $157(34.6)$ & 0.029 \\
\hline ARB & 948 (39.2) & $753(38.3)$ & $195(43.0)$ & 0.068 \\
\hline Aspirin & 2417 (99.9) & $1963(99.9)$ & $454(100.0)$ & 0.496 \\
\hline Clopidogrel & 2415 (99.8) & $1963(99.9)$ & $452(99.6)$ & 0.109 \\
\hline$\beta$-Blocker & 2199 (90.9) & $1780(90.6)$ & $419(92.3)$ & 0.255 \\
\hline Statins & 2366 (97.8) & $1922(97.8)$ & $444(97.8)$ & 0.985 \\
\hline Oral hypoglycemic agents & $437(18.1)$ & $314(16.0)$ & $123(27.1)$ & $<0.001$ \\
\hline
\end{tabular}


Table 1 Baseline clinical characteristics of the study population (Continued)

\begin{tabular}{|c|c|c|c|c|}
\hline & Total population, $n=2419$ & Without event, $n=1965$ & With event, $n=454$ & $P$ \\
\hline Insulin & $232(9.6)$ & $154(7.8)$ & $78(17.2)$ & $<0.001$ \\
\hline \multicolumn{5}{|l|}{ Angiographic data, n (\%) } \\
\hline Left main disease & $110(4.5)$ & $64(3.3)$ & $46(10.1)$ & $<0.001$ \\
\hline Multi-vessel disease & $1631(67.4)$ & $1225(62.3)$ & $406(89.4)$ & $<0.001$ \\
\hline Chronic total occlusion & $345(14.3)$ & $202(10.3)$ & $143(31.5)$ & $<0.001$ \\
\hline Diffuse lesion & $605(25.0)$ & $431(21.9)$ & $174(38.3)$ & $<0.001$ \\
\hline Bifurcation lesion & $492(20.3)$ & $368(18.7)$ & $124(27.3)$ & $<0.001$ \\
\hline Number of stents & $1.96 \pm 1.29$ & $1.87 \pm 1.14$ & $2.33 \pm 1.76$ & $<0.001$ \\
\hline
\end{tabular}

Bold values indicate statistically significant associations

$B M I$ Body mass index, SBP Systolic blood pressure, DBP Diastolic blood pressure, CAD Coronary artery disease, $M I$ Myocardial infarction, $P C I$ Percutaneous coronary intervention, $C A B G$ Coronary artery bypass grafting, $P A D$ Peripheral arterial disease, TGs Triglycerides, $T C$ Total cholesterol, $L D L-C$ Low-density lipoprotein cholesterol, HDL-C High-density lipoprotein cholesterol, RLP-C Remnant-like particle cholesterol, $h s$-CRP High-sensitivity C-reactive protein, eGFR Estimated glomerular filtration rate, FBG Fasting blood glucose, HbA1c Glycosylated hemoglobin A1c, LVEF Left ventricular ejection fraction, UA Unstable angina, NSTEMI NonST-segment elevation myocardial infarction, ACEI Angiotensin-converting enzyme inhibitor, ARB Angiotensin receptor blocker

Suppl. materials: Table S1) were constructed to evaluate the impact of estimated RLP-C on predicting composite and each component of the adverse events. After adjustment of the confounding factors, increased estimated RLP-C levels were consistently observed to be a significant predictor for adverse prognosis, despite regarding estimated RLP-C as a continuous or nominal variable (Table 2).

The addition of estimated RLP-C enhanced the AUC obtained from the baseline model adjusted for traditional risk factors including age, sex (female), smoking, hypertension, prior MI, prior PCI, eGFR, HbA1c, TC, HDL-C, LVEF, left main disease, and multi-vessel disease $(0.798$ for baseline model vs. 0.811 for baseline model + estimated RLP-C, $P$ for comparison <0.001) (Table 3, Fig. 3a). Moreover, adding estimated RLP-C to the baseline model improved the discriminative performance for prediction of adverse events (category-free NRI 0.084, $P=0.048$; IDI $0.017, P=0.030$ ) (Table 3).

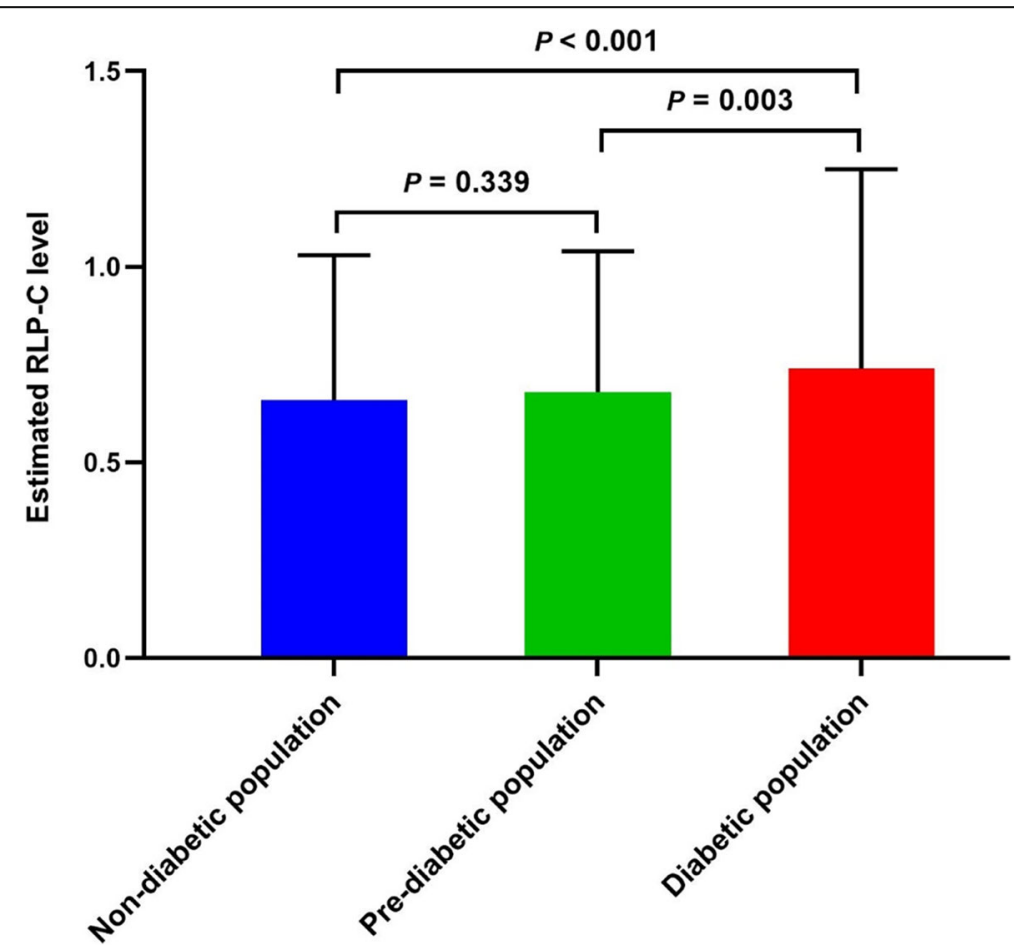

Fig. 1 Estimated RLP-C levels in different glycometabolic status. RLP-C, remnant-like particle cholesterol 


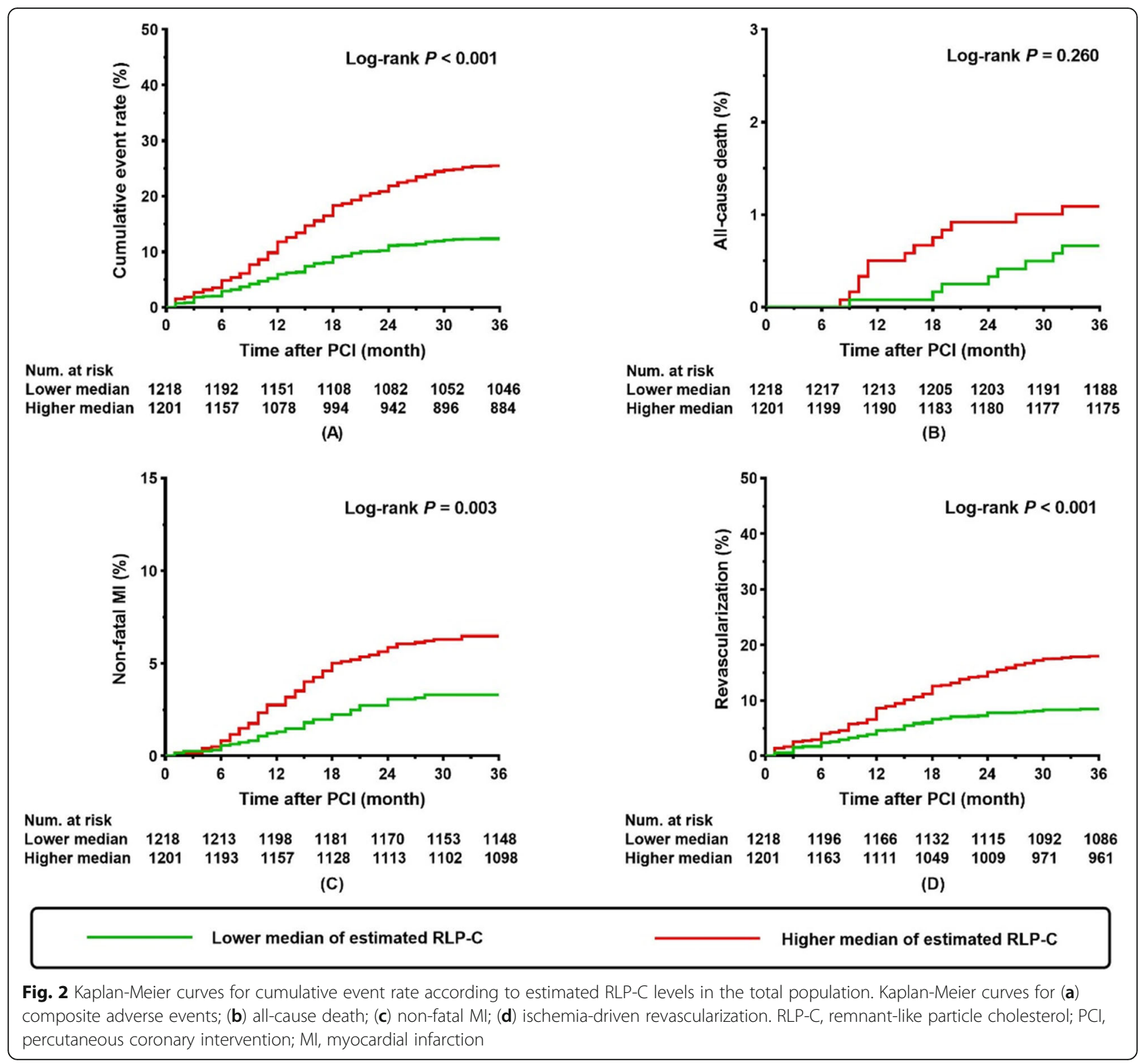

Table 2 Multiple Cox analysis on predictive value of estimated RLP-C for composite and each component of adverse events in the total population

\begin{tabular}{|c|c|c|c|c|c|c|c|c|}
\hline & \multicolumn{4}{|c|}{ As a nominal variable ${ }^{a}$} & \multicolumn{4}{|c|}{ As a continuous variable ${ }^{b}$} \\
\hline & $\beta$ & $\mathrm{HR}$ & $95 \% \mathrm{Cl}$ & $P$ & $\beta$ & $\mathrm{HR}$ & $95 \% \mathrm{Cl}$ & $P$ \\
\hline Composite adverse events & 0.673 & 1.960 & $1.558-2.465$ & $<0.001$ & 0.256 & 1.291 & $1.119-1.490$ & $<0.001$ \\
\hline All-cause death & 0.792 & 2.207 & $0.612-7.959$ & 0.226 & 0.604 & 1.829 & $0.837-3.995$ & 0.130 \\
\hline Non-fatal Ml & 0.633 & 1.883 & $1.195-2.966$ & 0.006 & 0.285 & 1.330 & $1.002-1.764$ & 0.048 \\
\hline Ischemia-driven revascularization & 0.608 & 1.836 & $1.395-2.416$ & $<0.001$ & 0.189 & 1.208 & $1.016-1.438$ & 0.033 \\
\hline
\end{tabular}

Bold values indicate statistically significant associations

Multiple Cox analysis was adjusted for confounders that are significant $(P<0.05)$ in simple Cox analysis (details shown in Suppl. materials: Table S1)

$H R$ Hazard ratio, Cl Confidence interval, MI Myocardial infarction

${ }^{\text {a }}$ The HR was examined regarding the lower median of estimated RLP-C as reference

${ }^{b}$ The HR was examined by per 1-SD increase of estimated RLP-C 
Table 3 C-statistics for discrimination ability of the various predictive model for composite adverse events in the total population

\begin{tabular}{|c|c|c|c|c|c|c|c|}
\hline & $\mathrm{ROCC}$ & lysis & & Categ & & $|D|$ & \\
\hline & $A \cup C$ & $95 \% \mathrm{Cl}$ & P & index & $P$ & index & $P$ \\
\hline Baseline model $^{a}$ & 0.798 & $0.781-0.814$ & reference & - & reference & - & reference \\
\hline + estimated RLP-C & 0.811 & $0.795-0.826$ & $<0.001$ & 0.084 & 0.048 & 0.017 & 0.030 \\
\hline
\end{tabular}

Bold values indicate statistically significant associations

ROC Receiver operating characteristics, AUC Area under the curve, CI Confidence interval, NRI Net reclassification improvement, IDI Integrated discrimination improvement, $R L P$-C Remnant-like particle cholesterol

a Baseline model includes traditional risk factors: age, sex (female), smoking, hypertension, prior MI, prior PCl, eGFR, HbA1C, TC, HDL-C, LVEF, left main disease and multi-vessel disease
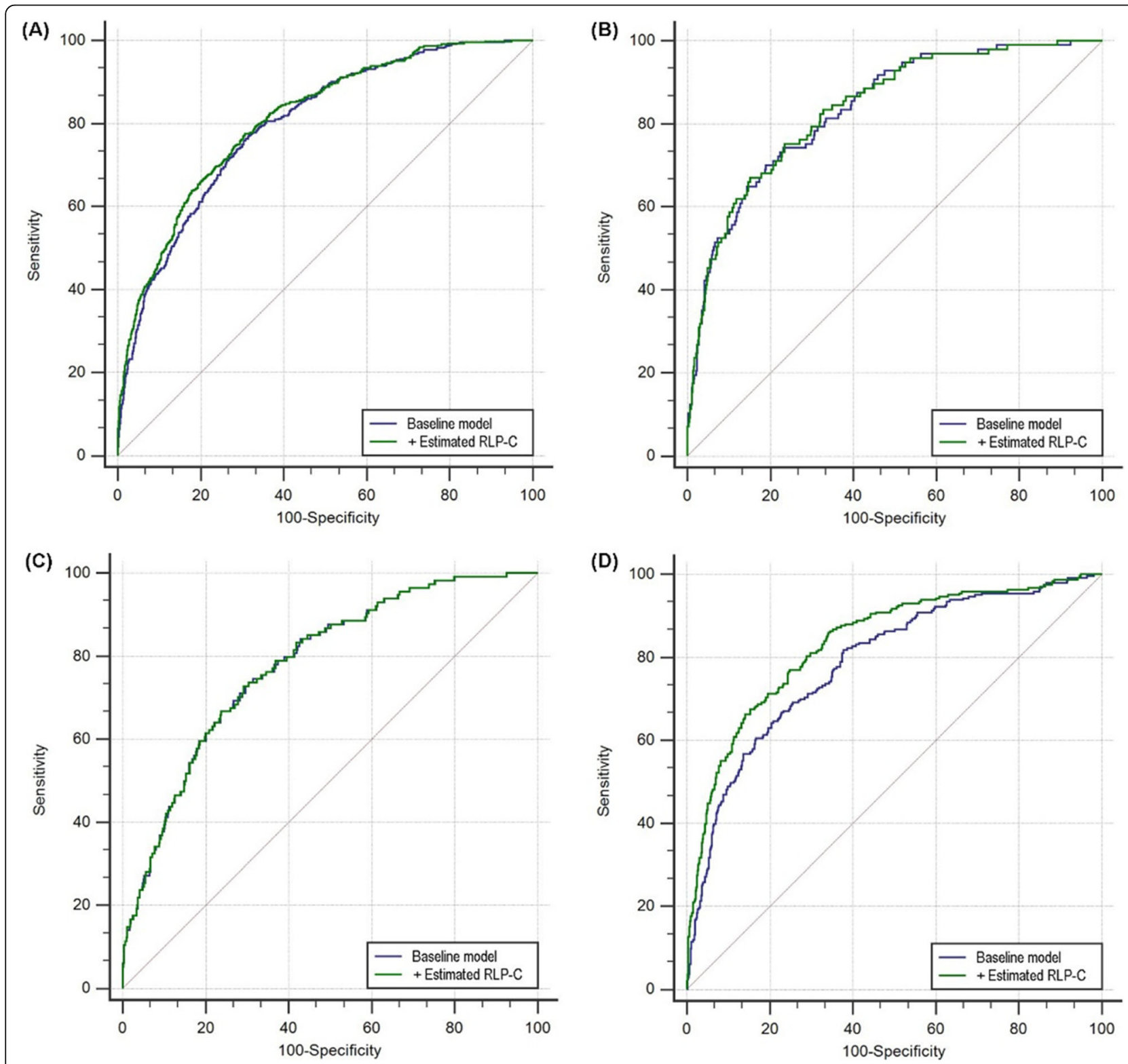

Fig. 3 ROC curve evaluating the predictive value of various models for composite adverse events in total population and subgroups. a Total population; b Non-diabetic population; c Pre-diabetic population; d Diabetic population. The baseline model includes traditional risk factors: age, sex (female), smoking, hypertension, prior MI, prior PCl, eGFR, HbA1c, TC, HDL-C, LVEF, left main disease and multi-vessel disease. RLP-C, remnantlike particle cholesterol 


\section{Predictive value of estimated RLP-C in subgroups with various glycometabolic status}

The predictive performance of estimated RLP-C was further evaluated in subgroups with various glycometabolic status [non-diabetic population $(n=926)$, pre-diabetic population $(n=645)$, diabetic population $(n=848)]$. Kaplan-Meier curves for the cumulative rate of the composite and each component of adverse events in various subgroups were summarized in Fig. 4. In patients with diabetes, those with a higher median of estimated RLP$\mathrm{C}$, as opposed to a lower median, exhibited a higher cumulative rate of composite adverse events, non-fatal MI, and ischemia-driven revascularization, (all $P<0.001$ ) (Fig. 4i-l). The difference was not found in pre-diabetic (Fig. 4e-h) and non-diabetic (Fig. 4a-d) patients.

In multiple Cox analysis, higher level of estimated RLP-C was shown to be prominently correlated to an increasing risk of adverse events in the diabetic population. However, estimated RLP-C failed to be a significant determinant of adverse events in the pre-diabetic and non-diabetic populations (Table 4).
The increased AUC resulting from adding estimated RLP-C to the baseline model was significant in the diabetic population $(0.788$ for baseline model vs. 0.836 for baseline model + estimated RLP-C, $P$ for comparison < 0.001) (Table 5, Fig. 3d). By contrast, the incremental effect on AUC was not seen in the pre-diabetic and nondiabetic populations (Table 5, Fig. $3 \mathrm{~b}$ and c). Furthermore, adding estimated RLP-C to the baseline model had a great improvement on the ability of predicting adverse events in the diabetic population (category-free NRI $0.155, P=0.010$; IDI $0.040, P<0.001$ ), but the additional effect was not found in the pre-diabetic and nondiabetic populations (Table 5).

\section{Discussion}

The current study confirmed an independent relationship between estimated RLP-C and recurrent adverse events in patients with NSTE-ACS undergoing PCI. Further subgroup analyses elucidated that estimated RLP-C showed a better predictive value in the diabetic population. However, estimated RLP-C failed to be an important

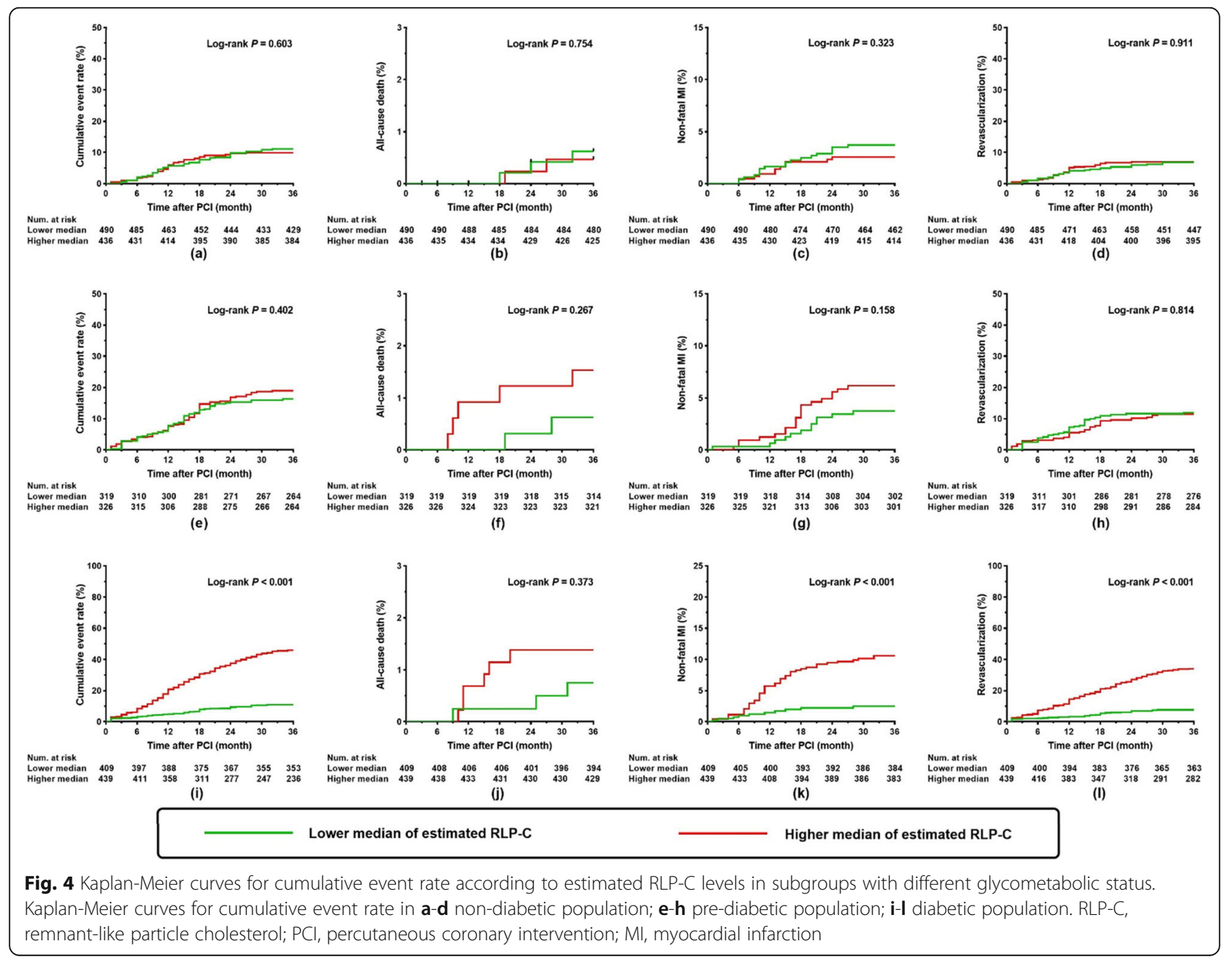


Table 4 Multiple Cox analysis on predictive value of estimated RLP-C for composite and each component of adverse event in subgroups with different glycometabolic status

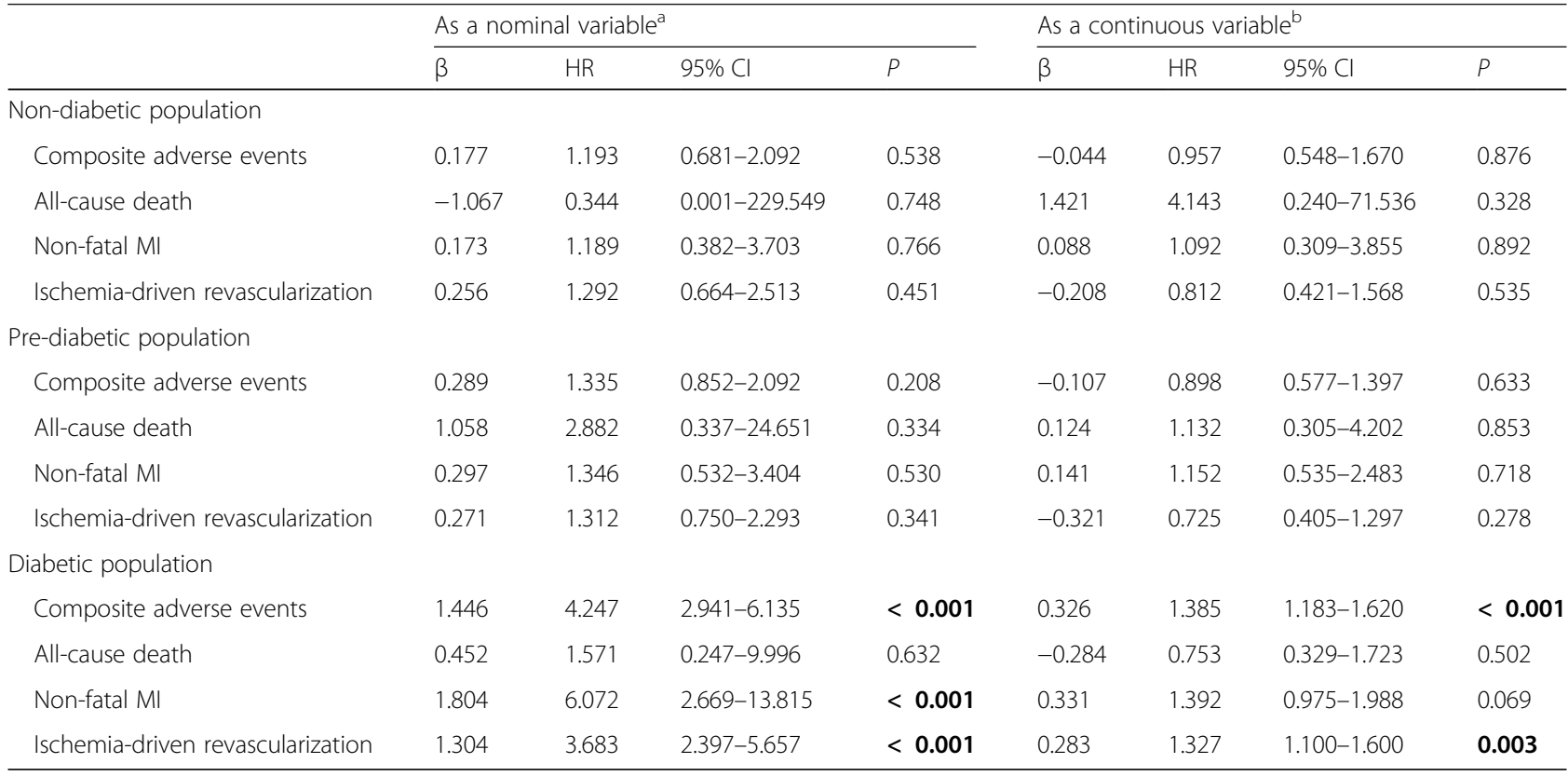

Bold values indicate statistically significant associations

Multiple Cox analysis was adjusted for confounders that are significant $(P<0.05)$ in simple Cox analysis (details shown in Suppl. materials: Table S1)

$H R$ Hazard ratio, $\mathrm{Cl}$ Confidence interval, $\mathrm{MI}$ Myocardial infarction

${ }^{a}$ The HR was examined regarding the lower median of estimated RLP-C as reference

${ }^{b}$ The HR was examined by per 1-SD increase of estimated RLP-C

determinant of worse outcomes in the pre-diabetic and non-diabetic populations. Adding estimated RLP-C to traditional risk factors exhibited a significant enhancement on the performance of predicting adverse events.

It has been widely demonstrated that LDL-C is one of the most significant risk indicators for ASCVD, and reduction of serum LDL-C levels with statins is a well- established therapy to reduce the ASCVD risk. However, many patients whose LDL-C levels are well controlled by statins continue to suffer recurrent cardiovascular events [3-7]. In recent years, factors related to obesity and metabolic syndrome, such as triglycerides rich lipoproteins (TRLs), have been considered as potential metabolismrelated risk factors for cardiovascular diseases and possible

Table 5 C-statistics for discrimination ability of the various predictive model for composite adverse events in subgroups with different glycometabolic status

\begin{tabular}{|c|c|c|c|c|c|c|c|}
\hline & \multicolumn{3}{|c|}{ ROC curve analysis } & \multicolumn{2}{|c|}{ Category-free NRI } & \multicolumn{2}{|l|}{$\mid \mathrm{DI}$} \\
\hline & $\mathrm{AUC}$ & $95 \% \mathrm{Cl}$ & $P$ & index & $P$ & index & $P$ \\
\hline \multicolumn{8}{|l|}{ Non-diabetic population } \\
\hline Baseline model ${ }^{a}$ & 0.836 & $0.810-0.859$ & reference & - & reference & - & reference \\
\hline + estimated RLP-C & 0.838 & $0.813-0.861$ & 0.311 & 0.022 & 0.517 & 0.002 & 0.169 \\
\hline \multicolumn{8}{|l|}{ Pre-diabetic population } \\
\hline Baseline model ${ }^{a}$ & 0.781 & $0.747-0.812$ & reference & - & reference & - & reference \\
\hline + estimated RLP-C & 0.781 & $0.747-0.812$ & 0.581 & 0.017 & 0.842 & 0.001 & 0.642 \\
\hline \multicolumn{8}{|l|}{ Diabetic population } \\
\hline Baseline model $^{a}$ & 0.788 & $0.759-0.815$ & reference & - & reference & - & reference \\
\hline + estimated RLP-C & 0.836 & $0.809-0.860$ & $<0.001$ & 0.155 & 0.010 & 0.040 & $<0.001$ \\
\hline
\end{tabular}

Bold values indicate statistically significant associations

ROC Receiver operating characteristics, AUC Area under the curve, Cl Confidence interval, NRI Net Reclassification improvement, IDI Integrated discrimination improvement, RLP-C Remnant-like particle cholesterol

aBaseline model includes traditional risk factors: age, sex (female), smoking, hypertension, prior Ml, prior PCl, eGFR, HbA1C, TC, HDL-C, LVEF, left main disease and multi-vessel disease 
cause of residual risks other than LDL-C. As the cholesterol component of the subset of TRLs, RLP-C has been demonstrated to be a causal risk factor of coronary artery disease (CAD) [23-25]. Previous studies also revealed that higher RLP-C levels showed favorable predictive value for adverse prognosis, either in those with stable CAD or ACS, regardless of the baseline treatment of statins and level of LDL-C [12, 26-29].

The relationship between RLP-C and plaque characteristics of coronary arteries including plaque burden, composition, and vulnerability has been disclosed by certain studies. Lina et al. revealed that RLP-C were significantly associated with plaque burden evaluated by coronary computed tomography angiography (CCTA), independent of the baseline levels of LDL-C [30]. Study from Puri et al. showed that non-HDL-C levels, which is highly correlated with RLP-C, were closely associated with the progression and regression of atherosclerotic plaque burden assessed by intravascular ultrasound, regardless of LDL-C levels [31]. Findings from Matsuo et al. revealed that in statin-treated patients, RLP-C levels, as opposed to LDL-C levels, were strongly associated with plaque vulnerability evaluated by the proportion of plaque necrosis [32]. These findings provide important confirmation and interpretation of results from previous clinical studies, suggesting that RLP-C is an important indicator of coronary atherosclerosis. Additionally, this correlation between RLP-C and plaque characteristics was observed in patients with an optimal level of LDL-C, indicating that RLP-C may be a residual risk factor on the basis of statin treatment.

In this study, the LDL-C level did not show a predictive value for poor prognosis, which was consistent with previous studies $[5,13,29]$. The underlying causes can be complex. Firstly, most participants that were enrolled in the present study underwent statin therapy, whose lipidlowering effects in conjunction with other effects may have potential impacts on the association of LDL-C levels with adverse events. Moreover, patients with complex coronary lesions or clinical conditions may be inclined to receive more intensive lipid-lowering therapy. Such treatment selection bias or so-called "confounding by indication" may have a certain influence on the predictive ability of LDL-C. Additionally this may lead to a paradox phenomenon, such as the phenomenon present in current study that the use of ACEI could predict adverse events. The present study revealed that the estimated RLP-C remained a predictor of adverse prognosis despite the probable influence of statin treatment, which indicates that the estimated RLP-C may have greater atherogenicity than other serum lipid parameters. TGs, TC, and HDL-C lost their predictive value in multiple Cox analysis, which may partly be attributed to the strong correlation between them and the estimated RLP-C.
Results from former studies have revealed that the impact of RLP-C seems to be more prominent in patients with metabolic syndromes or type 2 diabetes [12-16]. The current study also revealed that the predictive value of estimated RLP-C is significant only in patients with diabetes, which indicates that there is significant interaction between glycometabolic status and estimated RLP-C on risk prediction. Diabetic patients have more complex lipid metabolism disorders than non-diabetic patients characterized by increased TGs and decreased HDL-C [33]. Therefore, lipid-metabolic indicators except for LDL-C may also have certain impacts on the cardiovascular risk of diabetic patients. Previous studies have proven that hypertriglyceridemia and high TRLs are important predictors for CAD $[2,4,9]$. As the major carrier of TGs, TRLs binds to the arterial endothelium, where TGs are hydrolyzed by lipoprotein lipase, finally resulting in the generation of remnant lipoproteins. Thus, the level of TGs is closely related to the cholesterol component of remnant lipoproteins, namely, the RLP-C [34, 35]. The significant association of estimated RLP-C with TGs was also verified in the present study. Studies have also showed that RLP-C increased in patients with diabetes compared with those without [12, $26,35]$, which was consistent with the present study. These may all contribute to the significant prognostic impacts of RLP-C in patients with recognized diabetes.

Several pathophysiologic mechanisms may account for the relationship between RLP-C and the risk of recurrent adverse events. These include: (1) RLP-C can upregulate the expression of proinflammatory cytokines, which facilitate the monocytes moving into the arterial wall [36]; (2) RLP-C increases the generation of tissue factors (TF), which is essential for the formation of thrombus in vessels [36]; (3) There is evidence that RLP-C can enhance the aggregation of platelets [37]; (4) RLP-C promotes the propagation of smooth muscle cells that is independent from the impact of oxidative stress [38]; (5) RLP-C is causally related to low-grade inflammation, with a nearly triple increase in CRP for every $1 \mathrm{mmol} / \mathrm{L}$ increase in RLP-C [39]; (6) RLP-C was demonstrated to be an indicator of endothelial vasomotor dysfunction $[16,40]$; (7) High concentrations of RLP-C were proven to be correlated to inflammation in the arterial wall in cases of intimal damage [41]. The pro-inflammatory and proatherothrombotic roles of RLP-C listed above may be the explanation for the association between RLP-C and cardiovascular disease.

Studies have shown that less than a quarter of patients exhibited an LDL-C level below the guidelinerecommended target $[28,42]$. This discordance between target value and clinical practice is often called "treatment gap", which is a prevalent problem in the real world. In this context, while regarding LDL-C as the 
major target, the exploration of residual risk factors, such as RLP-C, can also provide complementary therapeutic strategies for reducing cardiovascular risk. Previous reports showed that lipid-lowering agents, such as fibrates, ezetimibe, and statins, as well as diet adaptation, proper aerobic exercise, and obesity reduction, may effectively decrease RLP-C levels to varying degrees [26, 43, 44], thus enabling RLP-C as a therapeutic target. Clinical trials of non-statin, lipid-lowering treatments have shown significant benefit in reducing residual risk, but none have specifically targeted RLP-C. Studies have demonstrated that omega-3 fatty acid derivatives [45] and antisense oligonucleotide to apolipoprotein C-III [46] have the potential to reduce TRLs significantly and provide useful tools for answering this question. In the JELIS study, eicosapentaenoic acid (an omega-3 fatty acid derivative) combined with low-dose statins reduced triglycerides by about $5 \%$ and coronary events by $19 \%$ compared to low-dose statins alone [47]. Furthermore, novel agents like inhibitors of apolipoprotein C-III and antibodies to PCSK9 were also proven to have promising results $[48,49]$. Nowadays, the pattern of targeting LDL$\mathrm{C}$ alone has changed, with recent guidelines highlighting the important role of non-HDL-C, which includes RLP$\mathrm{C}$, on the pathogenesis of atherosclerosis and thus its availability as an additional therapeutic target [11]. It is necessary to develop new therapies targeting RLP-C and conduct randomized trials evaluating whether lowering RLP-C levels can regulate plaque morphology and reduce the residual risk for cardiovascular outcomes.

\section{Study strengths and limitations}

This observational cohort study expanded the relationship between estimated RLP-C and poor outcomes to a population diagnosed with NSTE-ACS and received PCI treatment. The major strengths of the present study were the large number of enrolled subjects and the long follow-up period. Additionally, the prognostic impact of estimated RLP-C was evaluated in patients with differing glycometabolic status. There are some limitations in the present study: (1) In the fasting state, VLDL remnants are the primary constituent of circulating remnants, so the contribution of chylomicron remnants may have been underestimated [50]. (2) Although potentially not as accurate as direct measurement, especially among patients with elevated TGs [51], estimated RLP-C as used in the present study is easy to calculate by using routine lipid profiles and requires no additional expense. (3) The information about the type and dosage of statins, as well as other non-statin lipid-lowering agents is relatively scarce. (4) Finally, although sequential surveillance may provide more information, only baseline lipid profiles were obtained in the current study.

\section{Conclusions}

Estimated RLP-C is significantly associated with the recurrent adverse events in patients with diabetes and NSTE-ACS treated with PCI, as opposed to in the subgroup of pre-diabetic and non-diabetic populations. Adding estimated RLP-C to traditional risk factors significantly promotes the predictive performance for adverse events, especially in diabetic patients. The current study indicated that the evaluation of estimated RLP-C is important, not only for evaluating the risk of adverse prognosis, but also for tailoring treatment to prevent impending cardiovascular events in specific populations, such as diabetic patients. Further studies investigating whether appropriate therapeutic strategies targeting estimated RLP-C levels can improve the prognosis of CAD patients are needed to be proceeded.

\section{Supplementary information}

Supplementary information accompanies this paper at https://doi.org/10. 1186/s12944-020-01355-y.

Additional file 1: Table S1. Simple and multiple Cox analysis for composite adverse events (variates that are not significant in simple Cox analysis are not listed).

\section{Abbreviations}

ASCVD: Atherosclerotic cardiovascular disease; LDL-C: Low-density lipoprotein cholesterol; ACS: Acute coronary syndrome; RLP-C: Remnant-like particle cholesterol; non-HDL-C: Non-high-density lipoprotein cholesterol; NSTE-ACS: Non-ST-segment elevation acute coronary syndrome;

$\mathrm{PCl}$ : Percutaneous coronary intervention; HbA1c: Glycosylated hemoglobin A1c; TGs: Triglycerides; TC: Total cholesterol; HDL-C: High-density lipoprotein cholesterol; eGFR: Estimated glomerular filtration rate; MI: Myocardial infarction; HR: Hazard ratio; Cl: Confidence intervals; SD: Standard deviation; ROC: Receiver-operating characteristic; BMI: Body mass index; SBP: Systolic blood pressure; hs-CRP: High-sensitivity C-reactive protein; FBG: Fasting blood glucose; LVEF: Left ventricular ejection fraction; AUC: Area under the curve; NRI: Net reclassification improvement; IDI: Integrated discrimination improvement; TRLs: Triglycerides rich lipoproteins; CAD: Coronary artery disease; ACEl: Angiotensin-converting enzyme inhibitors; VLDL: Very-lowdensity lipoprotein

\section{Acknowledgements}

Not applicable.

\section{Authors' contributions}

QZ (first author) and TYZ made substantial contributions to study design, data collection, data analysis, and manuscript writing. YJZ (corresponding author) made substantial contributions to study design and intellectual direction. They contributed equally to this work. YJC, YM, YKX, JQY made contributions to data collection and analysis. All authors read and approved the final manuscript.

\section{Funding}

This work was supported by the grant from National Key Research and Development Program of China (2017YFC0908800); Beijing Municipal Administration of Hospitals "Ascent Plan" (DFL20150601) and "Mission plan" (SML20180601); Beijing Municipal Health Commission "Project of Science and Technology Innovation Center" (PXM2019_026272_000006)

(PXM2019_026272_000005).

\section{Availability of data and materials}

The datasets generated and analyzed for this study are available from the corresponding author upon reasonable request. 


\section{Ethics approval and consent to participate}

Given the retrospective nature of the current study, the requirement for informed consent was waived. The study protocol was approved by the Clinical Research Ethics Committee of Beijing Anzhen Hospital, Capital Medical University.

\section{Consent for publication}

Not applicable.

\section{Competing interests}

The authors declare that they have no conflicts of interest.

\section{Author details}

'Department of Cardiology, Beijing Anzhen Hospital, Capital Medical University, Beijing 100029, China. ${ }^{2}$ Beijing Institute of Heart Lung and Blood Vessel Disease, Beijing Key Laboratory of Precision Medicine of Coronary Atherosclerotic Disease, Clinical center for coronary heart disease, Capital Medical University, Beijing 100029, China. ${ }^{3}$ Research Center for Coronary Heart Disease, Fuwai Hospital, National Center for Cardiovascular Diseases, Chinese Academy of Medical Sciences and Peking Union Medical College, Beijing 100037, China.

\section{Received: 25 June 2020 Accepted: 27 July 2020}

Published online: 31 July 2020

\section{References}

1. Fox KAA, Carruthers KF, Dunbar DR, Graham C, Manning JR, De Raedt H, et al. Underestimated and under-recognized: the late consequences of acute coronary syndrome (GRACE UK-Belgian study). Eur Heart J. 2010; 31(22):2755-64.

2. Schwartz GG, Abt M, Bao W, DeMicco D, Kallend D, Miller M, et al. Fasting triglycerides predict recurrent ischemic events in patients with acute coronary syndrome treated with statins. J Am Coll Cardiol. 2015;65(21): 2267-75.

3. Baigent C, Blackwell L, Emberson J, Holland LE, Reith C, Bhala N, et al. Efficacy and safety of more intensive lowering of LDL cholesterol: a metaanalysis of data from 170,000 participants in 26 randomised trials. Lancet. 2010;376(9753):1670-81.

4. Miller M, Cannon CP, Murphy SA, Qin J, Ray KK, Braunwald E. Impact of triglyceride levels beyond low-density lipoprotein cholesterol after acute coronary syndrome in the PROVE IT-TIMI 22 trial. J Am Coll Cardiol. 2008; 51(7):724-30.

5. Mora S, Wenger NK, DeMicco DA, Breazna A, Boekholdt SM, Arsenault BJ, et al. Determinants of residual risk in secondary prevention patients treated with high- versus low-dose statin therapy. Circulation. 2012;125(16):1979-87.

6. Sabatine MS, Giugliano RP, Keech AC, Honarpour N, Wiviott SD, Murphy SA, et al. Evolocumab and clinical outcomes in patients with cardiovascular disease. N Engl J Med. 2017;376(18):1713-22.

7. Cannon CP, Blazing MA, Giugliano RP, McCagg A, White JA, Theroux P, et al. Ezetimibe added to statin therapy after acute coronary syndromes. N Engl J Med. 2015;372(25):2387-97.

8. Miller M, Ginsberg HN, Schaefer EJ. Relative atherogenicity and predictive value of non-high-density lipoprotein cholesterol for coronary heart disease. Am J Cardiol. 2008;101(7):1003-8.

9. Khetarpal SA, Rader DJ. Triglyceride-rich lipoproteins and coronary artery disease risk: new insights from human genetics. Arterioscler Thromb Vasc Biol. 2015;35(2):e3-9.

10. Chapman MJ, Ginsberg HN, Amarenco P, Andreotti F, Borén J, Catapano AL, et al. Triglyceride-rich lipoproteins and high-density lipoprotein cholesterol in patients at high risk of cardiovascular disease: evidence and guidance for management. Eur Heart J. 2011;32(11):1345-61.

11. Mach F, Baigent C, Catapano AL, Koskinas KC, Casula M, Badimon L, et al. 2019 ESC/EAS guidelines for the management of dyslipidaemias: lipid modification to reduce cardiovascular risk. Eur Heart J. 2020;41(1):111-88.

12. Fukushima H, Sugiyama S, Honda O, Koide S, Nakamura S, Sakamoto T, et al. Prognostic value of remnant-like lipoprotein particle levels in patients with coronary artery disease and type ii diabetes mellitus. J Am Coll Cardiol. 2004:43(12):2219-24

13. Nguyen SV, Nakamura T, Uematsu M, Fujioka D, Watanabe K, Watanabe Y, et al. Remnant lipoproteinemia predicts cardiovascular events in patients with type 2 diabetes and chronic kidney disease. J Cardiol. 2017;69(3):529-35.
14. Qin Z, Zhou K, Li Y, Wang J, Cheng W, Hu C, et al. Remnant lipoproteins play an important role of in-stent restenosis in type 2 diabetes undergoing percutaneous coronary intervention: a single-Centre observational cohort study. Cardiovasc Diabetol. 2019;18(1):11.

15. Nakamura T, Obata JE, Takano H, Kawabata K, Sano K, Kobayashi T, et al. High serum levels of remnant lipoproteins predict ischemic stroke in patients with metabolic syndrome and mild carotid atherosclerosis. Atherosclerosis. 2009;202(1):234-40.

16. Nakamura T, Takano H, Umetani K, Kawabata K, Obata JE, Kitta Y, et al. Remnant lipoproteinemia is a risk factor for endothelial vasomotor dysfunction and coronary artery disease in metabolic syndrome. Atherosclerosis. 2005;181(2):321-7.

17. Roffi M, Patrono C, Collet J, Mueller C, Valgimigli M, Andreotti F, et al. 2015 ESC guidelines for the management of acute coronary syndromes in patients presenting without persistent ST-segment elevation. Eur Heart J. 2016;37(3):267-315.

18. Association American Diabetes. Diagnosis and classification of diabetes mellitus. Diabetes Care. 2014;37(Suppl 1):S81-90.

19. Expert Dyslipidemia Panel of the International Atherosclerosis Society Panel members. An international atherosclerosis society position paper: global recommendations for the management of dyslipidemia--full report. J Clin Lipidol. 2014;8(1):29-60.

20. Jacobson TA, Ito MK, Maki KC, Orringer CE, Bays HE, Jones PH, et al. National lipid association recommendations for patient-centered management of dyslipidemia: part 1--full report. J Clin Lipidol. 2015;9(2):129-69.

21. Levey AS, Coresh J, Greene T, Stevens LA, Zhang YL, Hendriksen S, et al. Using standardized serum creatinine values in the modification of diet in renal disease study equation for estimating glomerular filtration rate. Ann Intern Med. 2006;145(4):247-54.

22. Section of Interventional Cardiology of Chinese Society of Cardiology of Chinese Medical Association, Specialty Committee on Prevention and Treatment of Thrombosis of Chinese College of Cardiovascular Physicians, Editorial Board of Chinese Journal of Cardiology. Chinese guideline for percutaneous coronary intervention (2016). Zhonghua Xin Xue Guan Bing Za Zhi. 2016;44(5):382-400

23. Varbo A, Benn M, Tybjærg-Hansen A, Jørgensen AB, Frikke-Schmidt R, Nordestgaard BG. Remnant cholesterol as a causal risk factor for ischemic heart disease. J Am Coll Cardiol. 2013;61(4):427-36.

24. Jørgensen AB, Frikke-Schmidt R, West AS, Grande P, Nordestgaard BG, Tybjærg-Hansen A. Genetically elevated non-fasting triglycerides and calculated remnant cholesterol as causal risk factors for myocardial infarction. Eur Heart J. 2013;34(24):1826-33.

25. Joshi PH, Khokhar AA, Massaro JM, Lirette ST, Griswold ME, Martin SS, et al. Remnant lipoprotein cholesterol and incident coronary heart disease: the Jackson heart and Framingham offspring cohort studies. J Am Heart Assoc. 2016;5(5):e2765.

26. Kugiyama K, Doi H, Takazoe K, Kawano H, Soejima H, Mizuno Y, et al. Remnant lipoprotein levels in fasting serum predict coronary events in patients with coronary artery disease. Circulation. 1999:99(22):2858-60.

27. Jepsen AM, Langsted A, Varbo A, Bang LE, Kamstrup PR, Nordestgaard BG. Increased remnant cholesterol explains part of residual risk of all-cause mortality in 5414 patients with ischemic heart disease. Clin Chem. 2016; 62(4):593-604.

28. Nguyen SV, Nakamura T, Kugiyama K. High remnant lipoprotein predicts recurrent cardiovascular events on statin treatment after acute coronary syndrome. Circ J. 2014;78(10):2492-500.

29. Fujihara Y, Nakamura T, Horikoshi T, Obata JE, Fujioka D, Watanabe Y, et al. Remnant lipoproteins are residual risk factor for future cardiovascular events in patients with stable coronary artery disease and on-statin low-density lipoprotein cholesterol levels <70 mg/dL. Circ J. 2019;83(6):1302-8.

30. Lin A, Nerlekar N, Rajagopalan A, Yuvaraj J, Modi R, Mirzaee S, et al. Remnant cholesterol and coronary atherosclerotic plaque burden assessed by computed tomography coronary angiography. Atherosclerosis. 2019;284:24-30.

31. Puri R, Nissen SE, Shao M, Elshazly MB, Kataoka Y, Kapadia SR, et al. Non-HDL cholesterol and triglycerides: implications for coronary atheroma progression and clinical events. Arterioscler Thromb Vasc Biol. 2016;36(11):2220-8.

32. Matsuo N, Matsuoka T, Onishi S, Yamamoto H, Kato A, Makino Y, et al. Impact of remnant lipoprotein on coronary plaque components. J Atheroscler Thromb. 2015;22(8):783-95.

33. Krauss RM. Lipids and lipoproteins in patients with type 2 diabetes. Diabetes Care. 2004;27(6):1496-504. 
34. Nordestgaard BG. Triglyceride-rich lipoproteins and atherosclerotic cardiovascular disease: new insights from epidemiology, genetics, and biology. Circ Res. 2016;118(4):547-63.

35. Goliasch G, Wiesbauer F, Blessberger H, Demyanets S, Wojta J, Huber K, et al. Premature myocardial infarction is strongly associated with increased levels of remnant cholesterol. J Clin Lipidol. 2015;9(6):801-6.

36. Doi H, Kugiyama K, Oka H, Sugiyama S, Ogata N, Koide Sl, et al. Remnant lipoproteins induce proatherothrombogenic molecules in endothelial cells through a redox-sensitive mechanism. Circulation. 2000;102(6):670-6.

37. Saniabadi AR, Umemura K, Shimoyama M, Adachi M, Nakano M, Nakashima M. Aggregation of human blood platelets by remnant like lipoprotein particles of plasma chylomicrons and very low density lipoproteins. Thromb Haemost. 1997;77(5):996-1001.

38. Zhao D, Letterman J, Schreiber BM. Beta-migrating very low density lipoprotein (beta VLDL) activates smooth muscle cell mitogen-activated protein (MAP) kinase via G protein-coupled receptor-mediated transactivation of the epidermal growth factor (EGF) receptor: effect of MAP kinase activation on beta VLDL plus EGF-induced cell proliferation. J Biol Chem. 2001;276(33):30579-88.

39. Varbo A, Benn M, Tybjærg-Hansen A, Nordestgaard BG. Elevated remnant cholesterol causes both low-grade inflammation and ischemic heart disease, whereas elevated low-density lipoprotein cholesterol causes ischemic heart disease without inflammation. Circulation. 2013;128(12): 1298-309.

40. Kugiyama K, Doi H, Motoyama T, Soejima H, Misumi K, Kawano H, et al. Association of remnant lipoprotein levels with impairment of endotheliumdependent vasomotor function in human coronary arteries. Circulation. 1998:97(25):2519-26.

41. Bernelot MS, Verweij SL, Schnitzler JG, Stiekema L, Bos M, Langsted A, et al. Remnant cholesterol elicits arterial wall inflammation and a multilevel cellular immune response in humans. Arterioscler Thromb Vasc Biol. 2017; 37(5):969-75.

42. Assmann G, Benecke H, Neiss A, Cullen P, Schulte H, Bestehorn K. Gap between guidelines and practice: attainment of treatment targets in patients with primary hypercholesterolemia starting statin therapy. Results of the 4 E-registry (efficacy calculation and measurement of cardiovascular and cerebrovascular events including Physicians' experience and evaluation). Eur J Cardiovasc Prev Rehabil. 2006;13(5):776-83.

43. Packard CJ. Determinants of achieved LDL cholesterol and "non-HDL" cholesterol in the management of dyslipidemias. Curr Cardiol Rep. 2018; 20(8):60.

44. Bozzetto L, Annuzzi G, Corte GD, Patti L, Cipriano P, Mangione A, et al. Ezetimibe beneficially influences fasting and postprandial triglyceride-rich lipoproteins in type 2 diabetes. Atherosclerosis. 2011;217(1):142-8.

45. Ballantyne CM, Braeckman RA, Soni PN. Icosapent ethyl for the treatment of hypertriglyceridemia. Expert Opin Pharmacother. 2013;14(10):1409-16

46. Graham MJ, Lee RG, Bell TR, Fu W, Mullick AE, Alexander VJ, et al. Antisense oligonucleotide inhibition of apolipoprotein C-III reduces plasma triglycerides in rodents, nonhuman primates, and humans. Circ Res. 2013; 112(11):1479-90.

47. Yokoyama M, Origasa H, Matsuzaki M, Matsuzawa Y, Saito Y, Ishikawa Y, et al. Effects of eicosapentaenoic acid on major coronary events in hypercholesterolaemic patients (JELIS): a randomised open-label, blinded endpoint analysis. Lancet. 2007;369(9567):1090-8.

48. TG and HDL Working Group of the Exome Sequencing Project, National Heart, Lung, and Blood Institute. Loss-of-function mutations in APOC3, triglycerides, and coronary disease. N Engl J Med. 2014;371(1):22-31.

49. Dijk W, Le May C, Cariou B. Beyond LDL: what role for PCSK9 in triglyceriderich lipoprotein metabolism? Trends Endocrinol Metab. 2018;29(6):420-34.

50. Nakamura K, Miyoshi T, Yunoki K, Ito H. Postprandial hyperlipidemia as a potential residual risk factor. J Cardiol. 2016:67(4):335-9.

51. Chen J, Kuang J, Tang X, Mao L, Guo X, Luo Q, et al. Comparison of calculated remnant lipoprotein cholesterol levels with levels directly measured by nuclear magnetic resonance. Lipids Health Dis. 2020;19(1):132.

\section{Publisher's Note}

Springer Nature remains neutral with regard to jurisdictional claims in published maps and institutional affiliations.

\section{Ready to submit your research? Choose BMC and benefit from:}

- fast, convenient online submission

- thorough peer review by experienced researchers in your field

- rapid publication on acceptance

- support for research data, including large and complex data types

- gold Open Access which fosters wider collaboration and increased citations

- maximum visibility for your research: over $100 \mathrm{M}$ website views per year

At BMC, research is always in progress.

Learn more biomedcentral.com/submissions 